LA-UR- $\quad 09-101354$

Approved for public release; distribution is unlimited.

Title:

Shock Induced Multi-mode Damage in Depleted Uranium

Author(s):

D.D. Koller, E.K. Cerreta, and G.T. Gray III

Intended for:

European Physical Journal

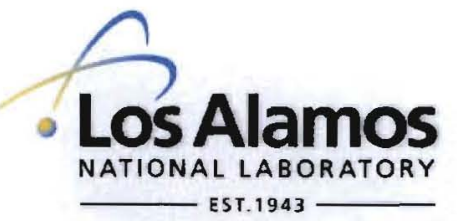

Los Alamos National Laboratory, an affirmative action/equal opportunity employer, is operated by the Los Alamos National Security, LLC for the National Nuclear Security Administration of the U.S. Department of Energy under contract DE-AC52-06NA25396. By acceptance of this article, the publisher recognizes that the U.S. Government retains a nonexclusive, royalty-free license to publish or reproduce the published form of this contribution, or to allow others to do so, for U.S. Government purposes. Los Alamos National Laboratory requests that the publisher identify this article as work performed under the auspices of the U.S. Department of Energy. Los Alamos National Laboratory strongly supports academic freedom and a researcher's right to publish; as an institution, however, the Laboratory does not endorse the viewpoint of a publication or guarantee its technical correctness.

Form $836(7 / 06)$ 


\title{
Shock Induced Multi-mode Damage in Depleted Uranium ${ }^{a}$
}

\author{
D. D. Koller, E.K. Cerreta, and G.T. Gray III \\ Los Alamos National Laboratory, Los Alamos, NM 87545
}

\begin{abstract}
Recent dynamic damage studies on depleted uranium samples have revealed mixed mode failure mechanisms leading to incipient cracking as well as ductile failure processes. Results show that delamination of inclusions upon compression may provide nucleation sites for damage initiation in the form of crack tip production. However, under tension the material propagates cracks in a mixed shear localization and mode-I ductile tearing and cracking. Cracks tips appear to link up through regions of severe, shear dominated plastic flow. Shock recovery experiments were conducted on a $50 \mathrm{~mm}$ single stage light gas gun. Serial metallographic sectioning was conducted on the recovered samples to characterize the bulk response of the sample. Experiments show delaminated inclusions due to uniaxial compression without damage propagation. Further results show the propagation of the damage through tensile loading to the incipient state, illustrating ductile processes coupled with mixed mode-l tensile ductile tearing, shear localization, and mode-l cracking in depleted uranium.
\end{abstract}

\section{INTRODUCTION}

Uranium is a low symmetry engineering material used in many nuclear fuel applications. Depleted uranium (DU) is natural uranium with the percentage of $U^{235}$ isotope lowered to below $0.2 \%$ and has use in many non-nuclear applications such as inertial guidance devices, gyro compasses, counterweights for aircraft control surfaces, ballast for missile reentry vehicles, kinetic energy penetrators, and radiation shielding [1]. It is a high density material $\left(19.1 \mathrm{~g} / \mathrm{cm}^{3}\right)$ and is easily cast and formed. The most common form of uranium is the $U^{238}$ isotope in the low temperature $\alpha$ orthorhombic phase. This phase is considered to be ductile, but its ductility is strongly dependent on the impurity content [2]. Impurities such as carbon and other uranium isotopes are common in uranium samples. The low solubility of impurities in uranium leads to second phase particles, such as carbides, that decrease the ductility of the material.

Shock compression techniques are often used to investigate tensile damage in metals subjected to extreme loading conditions. Dynamic tensile damage occurs when rarefaction (expansion) waves within a material interact in such a manner as to produce tensile stresses in excess of the threshold required for damage initiation [3]. If the tension is of sufficient amplitude, damage will accrue and thereafter a new interface will form within the material, creating a "spall layer". Plate impact experiments offer several advantages to the investigation of dynamic tensile damage over other techniques including the ability to maintain truly global one-dimensional, planar strain loading conditions for the duration of the experiment, albeit on a local level once damage evolution begins the local stress state is three dimensional, and the ability to recover the material for post-experiment metallurgical analysis.

Evidence that a spall layer has been created in a plate impact experiment is often obtained using a velocity interferometer system for any reflector (VISAR) [4] and has been the subject of numerous studies $[5,6]$. In general, the free surface velocity profile obtained using VISAR shows ringing consistent with wave reflections within a layer thinner than the original sample. If the tension is not of sufficient amplitude to form a completely separate layer, a region of significant damage, termed "incipient spallation, - consisting of a localized band of voids and or cracks, for example - may still exist. This region may be of significantly lower impedance than the undamaged region and may produce a velocity profile similar or even identical to that obtained from the completely separated

${ }^{\text {a }}$ LA-UR-08-06705 
layer. Insight into this type of ambiguity can be found through post experiment sample recovery and quantitative material damage evolution analysis. Early studies into the spall response of DU have been conducted $[7,8]$ in an effort to better understand the cumulative dynamic evolution response of this material.

\section{EXPERIMENTAL TECHNIQUES}

\subsection{Plate Impact Experiments}

Plate impact experiments were conducted using a smooth bore $50 \mathrm{~mm}$ diameter light gas gun. Sapphire impactors were mounted to the front of aluminum sabots and accelerated down the barrel to velocities of approximately $300 \mathrm{~m} / \mathrm{s}$. Spall is generated in the sample when rarefaction waves generated at the back of the impactor travel into the sample and interact (in the bulk of the target sample) with the rarefaction waves generated at the free surface of the sample material. When this interaction begins, a region of tension is formed which leads to damage evolution and if the magnitude of the impulse exceeds the fracture resistance of the material, ultimately spall in the material [9].

To ensure that recovered samples have been subjected only to a known uniaxial loading/unloading history, it is important to prevent the sample from undergoing any significant radial release. Typically, this is accomplished by placing rings around the sample made of the same material referred to as "momentum trapping" rings. If properly designed, the rarefaction release wave relieves the stress to zero in the sample uniaxially and the sample separates from the rings before edge release waves reach it. This technique commonly referred to as momentum trapping [10], has proven effective in preserving uniaxial loading/unloading in numerous recovery and non-recovery experiments [11-14]. Finite difference calculations by Stevens and Jones [15] demonstrated that if the momentum trapping rings are not properly designed, the plastic work on the sample from radial release waves can be up to a factor of ten higher than that resulting from uniaxial loading/unloading only. Furthermore, a diameter to thickness ratio of greater than 7 to 1 is needed to reduce the radial release effects to acceptably low levels. In the review by Gray [10], a simple centered flow condition calculation is used to determine the approximate minimum width for the momentum trapping rings.

Table 1. Experimental Parameters

\begin{tabular}{lllll}
\hline Shot number & Impactor & $\begin{array}{l}\text { Impactor } \\
\text { thickness }(\mathrm{mm})\end{array}$ & $\begin{array}{l}\text { Target thickness } \\
(\mathrm{mm})\end{array}$ & $\begin{array}{l}\text { Impact velocity } \\
\text { (mm/us) }\end{array}$ \\
\hline \hline $56-08-15$ & z-cut Quartz & 4.076 & 2.463 & 0.183 \\
$56-08-16$ & z-cut Quartz & 3.063 & 2.511 & 0.287 \\
$56-08-23$ & z-cut Quartz & 3.055 & 2.463 & 0.270 \\
\hline \hline
\end{tabular}

\subsection{Sample Preparation}

The material used for this investigation was a depleted-uranium (DU) plate material. The annealed DU possessed an equiaxed grain structure with an average grain size of $18 \mu \mathrm{m}$. The analyzed chemical composition of this depleted uranium (wt. pct.) was: $14 \mathrm{ppm} \mathrm{Al}, 40 \mathrm{ppm} \mathrm{C},<10 \mathrm{ppm} \mathrm{Ca}$, $<10 \mathrm{ppm} \mathrm{Fe},<2 / \mathrm{ppm} \mathrm{Mg}, 4 \mathrm{ppm} \mathrm{Mn}, 11 \mathrm{ppm} \mathrm{Ni},<10 \mathrm{ppm} \mathrm{Si}$, and bal. U. The initial plate texture, quantified usingelectron back scattered diffraction (EBSD), consisted of a strong [001] component in the plate through-thickness (TT) direction (max. 3.8 times random) with moderate texture asymmetries within the plane of the plate.

Using the general relationship given by Gray et al [10], only a single momentum trapping ring approximately $12 \mathrm{~mm}$ wide is needed to mitigate edge waves. The experimental facility used at Los Alamos National Laboratory allows for samples up to $38 \mathrm{~mm}$ in diameter. Since the sample diameter 
in these experiments is only $15 \mathrm{~mm}$ for recovery, two additional rings were used to decrease the likelihood that edge waves would reach the sample. Furthermore, the sample and inner ring were machined with a $7^{\circ}$ taper to ensure that the sample will separate from the rings. This taper, and the geometry of the rings, is illustrated in Fig.1.

After press fitting the sample and rings, the assembly was lapped flat and parallel to better than $5 \mu \mathrm{m}$ and glued into a target plate. Several diagnostic pins were positioned around the perimeter of the target assembly to measure impactor velocity and tilt and to trigger other diagnostic equipment. After

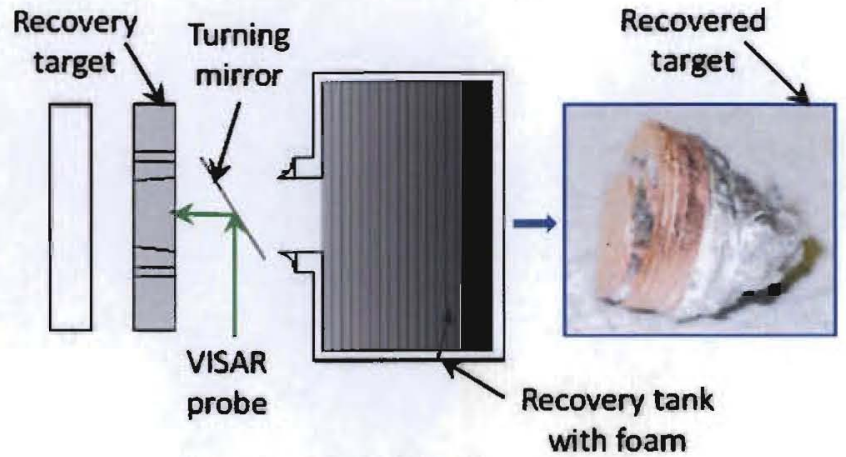

Figure 1. Experimental configuration. assembly, the parallelism of the sample to the target plate and the height of each pin with respect to the target plate are measured. Final sample dimensions were nominally $38 \mathrm{~mm}$ DIA X $2.5 \mathrm{~mm}$ thick right circular cylinders. Diagnostic velocimetry probes were mounted at $90^{\circ}$ off the shock direction axis to prevent impact with the probe prior to recovery. A mylar mirror with an $8 \mathrm{um} \mathrm{Ag}$ coating was used to direct the laser at the free surface of the sample. A drawing of the experimental setup is shown in Fig. 1. Photon Doppler Velocimetry (PDV) was used to obtain velocimetry information over a spot size of approximately $0.5 \mathrm{~mm}$. This technique is described elsewhere [16]. Experimental parameters are listed in Table 1.

\subsection{Post-experiment Metallurgical Analysis}

The damage evolution and post-shock microstructures of each of the samples following spallation loading were characterized using optical microscopy as well as scanning electron microscopy. All specimens were sectioned parallel to the shock loading axis along the centerline of the specimen, mechanically ground and polished, electro-chemically polished, and subsequently chemically etched. Optical microscopy was utilized to reveal the extent of the damage in the direction of loading.

Fracture surfaces, within the incipiently-spalled specimens, were observed using an Inspect $F$ Scanning Electron Microscope (SEM). To view incipiently-spalled fracture surfaces, specimens were mounted and polished such that the loading direction was the normal to the surface being examined. Then these specimens were ground down to the fracture plane for observation. This technique was successful in that it allowed for observation of the incipiently-spalled surfaces without disturbing the nature of these surfaces. However, the incipient nature of the fracture did not allow for bulk fracture surface observation but the few surfaces that were examined displayed principally intergranular fracture.

\section{RESULTS AND CONCLUSIONS}

Figure 2 shows the extracted $\left(\mathrm{u}_{\mathrm{p}} \mathrm{t} \mathrm{t}\right.$ data is extracted from the spectrogram obtained from the PDV diagnostic technique) velocimetry results for all experiments detailed

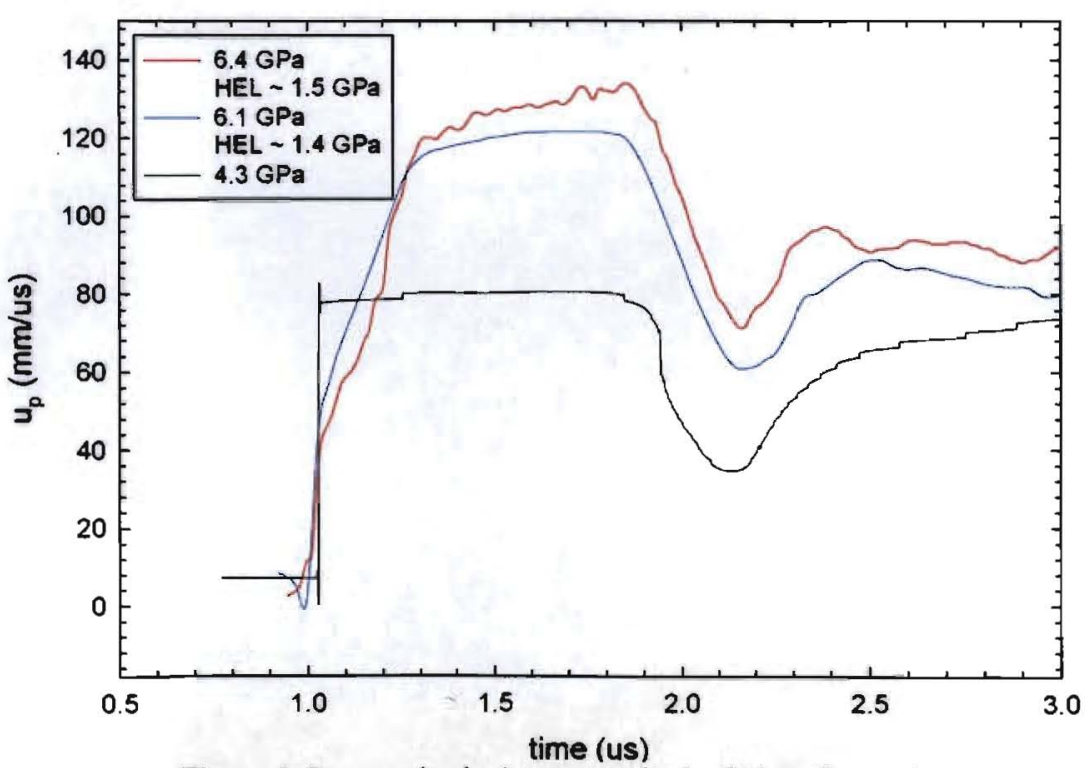

Figure 2. Extracted velocimetry results for DU spall experiments. 


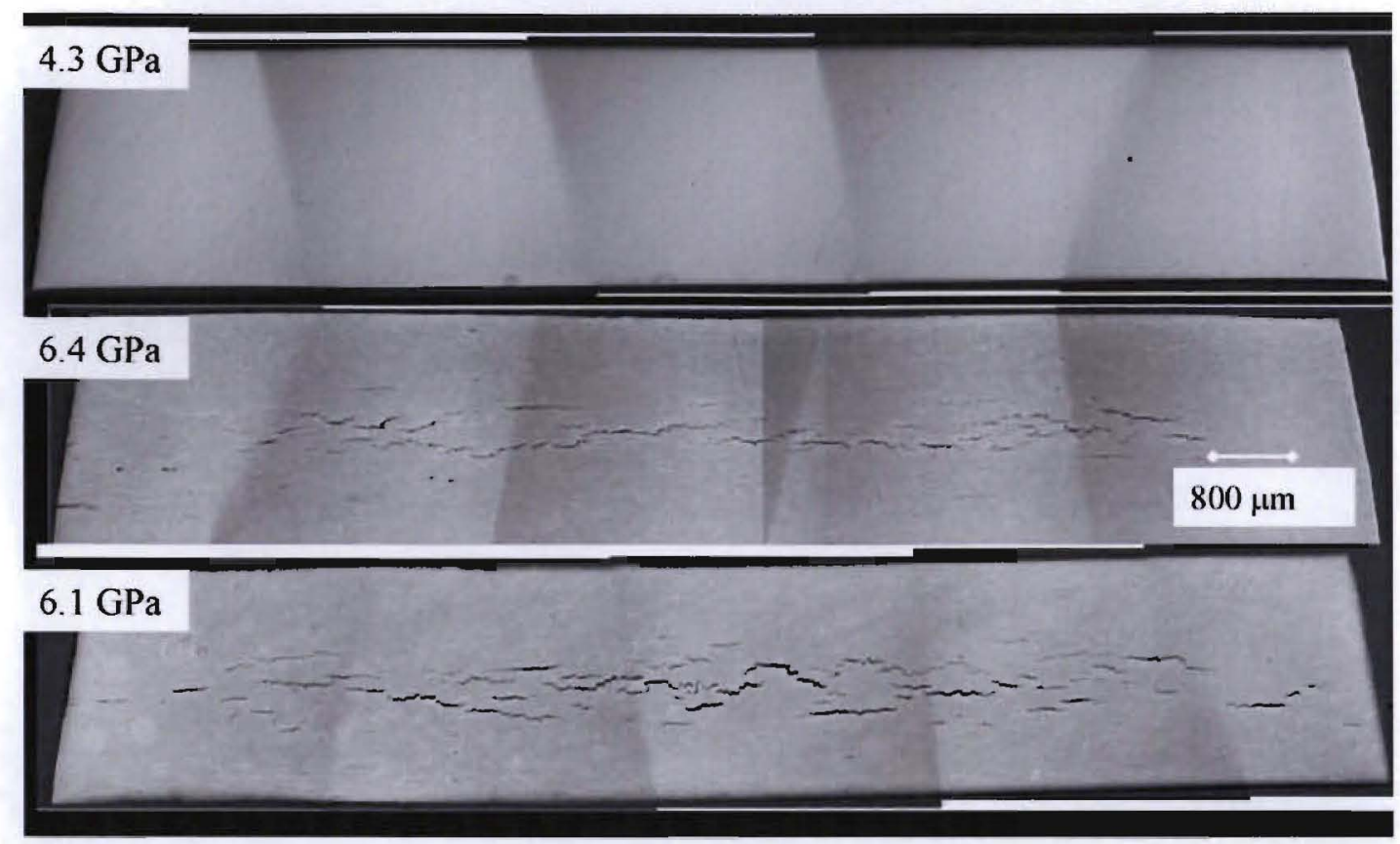

in Table 1. In the case of the $4.3 \mathrm{GPa}$ experiment, it appears that the specimen deformed elastically.

Figure 3. Macroscopic optical images of recovered DU samples showing a) delamination of inclusion through the bulk of the sample, b) and c) localized insipiently cracked regions.

This is indicated by the sharp initial first shock maintained through the sample thickness as well as no evidence of yielding in release. As the tensile region develops in this experiment, the DU compensates elastically showing a reacceleration to the peak state at the free surface due to the wave interaction.

At higher peak stresses, the HEL is observed. Yielding is apparent in the release with a strong pullback signal. The $6.4 \mathrm{GPa}$ experiment shows that the peak state is not a steady state, indicative of non-equilibrium conditions. This experiment is repeated at $6.1 \mathrm{GPa}$ to show repeatability. In this case, the velocimetry indicates a steady peak state as well as yielding.

Figure 3 shows the macroscopic optical images of the all experiments. The two high-pressure experimental samples (6.4 GPa and 6.1 GPa shown in Fig. $3 \mathrm{~b}$ and 3c) display a visible spall damage region in the form of lateral cracking centered at $1.2 \mathrm{~mm}$ from the free surface. At $4.3 \mathrm{GPa}$ (Fig. 3a) the sample does not show any significant damage beyond delamination of inclusions. Inclusions appear to delaminate through the entire bulk of the sample indicating that this is likely a result of the compression and/or rarefaction waves and not the tensile region that only forms in a discretely
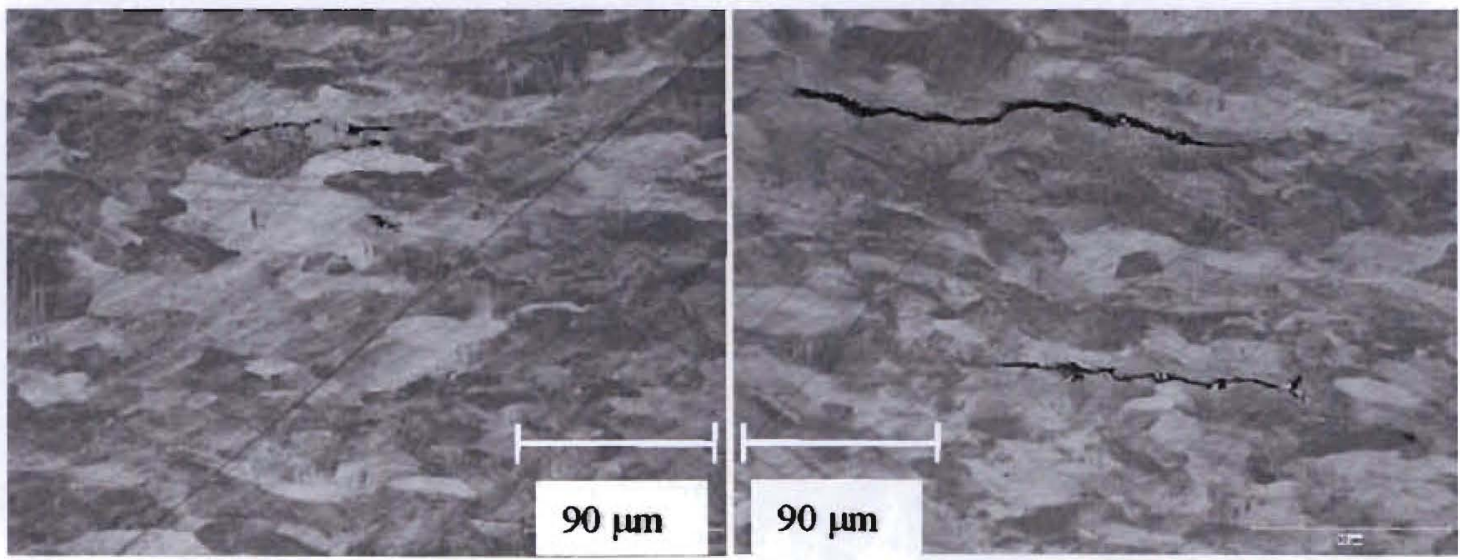

Figure 4. Detailed optical micrographs of $4.3 \mathrm{GPa}$ recovered DU sample showing crack initiation from delaminated inclusions. 
localized portion of the bulk. This delamination of the inclusions from the surrounding matrix is not surprising given the modulus and strength differences between the lattice and the oxides/carbides as
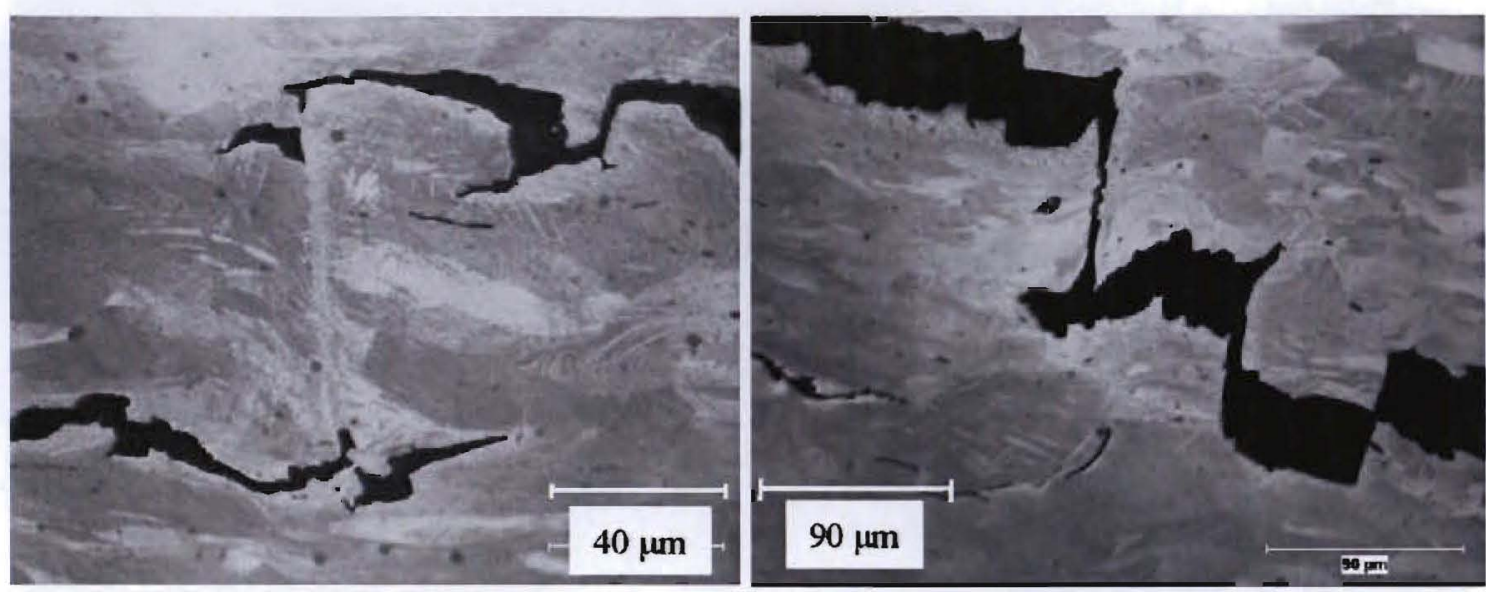

Figure 5. Detailed optical micrographs from $6 \mathrm{GPa}$ experiments showing a) unopened plastic flow or shear band between crack tips and b) opened plastic regions between crack tips.

plasticity sweeps through during the shock. Detailed micrographs of the $4.3 \mathrm{GPa}$ experiment are shown in Fig. 4. The microstructure is heavily twinned and cracks appear through the bulk of the sample initiating primarily from inclusion sites.

In the $6.4 \mathrm{GPa}$ experiment (Fig. 3b), the damaged region due to the spallation loading cycle is approximately $0.5 \mathrm{~mm}$ thick. The average crack area was $0.003 \mathrm{~mm}^{2}$ giving a total area fraction of the cracked region of $0.151 \mathrm{~mm}^{2}$ which accounts for $0.45 \%$ of the total area. In the $6.1 \mathrm{GPa}$ experiment (Fig. 3c), the damaged spall region is approximately $0.76 \mathrm{~mm}$ thick. The average crack area is $0.0004 \mathrm{~mm}^{2}$ giving a total area fraction of the cracked region of $0.331 \mathrm{~mm}^{2}$ which accounts for $0.97 \%$ of the total area. The difference in damaged volume is likely due to the difference in peak stress. The lower peak stress is a direct result of a lower impact velocity. In this condition of lower impact velocity expansion of rarefaction waves is enhanced for a consistent sample thickness. This produces a larger volume of material over which the rarefaction waves interact to produce tension.

Details of the damaged regions in the insipiently cracked samples are shown in Fig. 5. The microstructure is seen to be heavily twinned and although no macroscopic shear banding is observed, crack tips appear to be linked through regions of localized plastic flow (Fig. 5a). Plastic/ductile tearing damage appears to be limited to isolated regions and in some cases these regions open as well (Fig. 5b). Cracking appears to initiate from inclusion sites and approximately $50 \%$ clearly follow grain boundaries after initiation.

\section{Acknowledgments}

The authors would like the thanks Mark Byers and Ann Kelly for their help in making this study possible. This work was conducted under the auspices of the U.S. Department of Energy and the NNSA.

\section{References}

[1] CRC Handbook of Chemistry and Physics, eds. R. Weast and M. Astle, CRC Press, Boca Raton, FL (1981).

[2] Eckelmeyer, K.H., in Uranium and Uranium Alloys, ASM International, Materials Park, Ohio, 1991.

[3] T. Antoun, L. Seaman, D. Curran, G. Kanel, S. Razorenov, A. Utkin, Spall Fracture, Springer (2002) pg 26. 
[4] L.M. Barker and R.E. Hollenbach, J.A.P., 43, 4669 (1972).

[5] W.F. Hemsing, Review of Scientific Intruments, 50 pp 73-78 (1979).

[6] R.S. Hixson, G.T. Gray III, P.A. Rigg, L.B. Addessio and C.A. Yablinsky, Shock Compression of Condensed Matter, 2003, edited by M.D. Furnish, Y.M. Gupta, and J.W. Forbes, pg 469.

[7] Zurek, A.K., Embury, J.D., Kelly, A., Thissell, W.R., Gustavsen, R.L., Vorthman, J.E., Hixson, R.S., in Shock Compression of Condensed Matter, AIP, (1991).

[8] S. Cochran and D. Banner, J. Appl. Phys, Vol 48, No. 7, (1977).

[9] Jim Johnson 1981

[10] G.T. Gray III, Influence of Shock-Wave Deformation on the Structure/Property Behavior of Materials, in High Pressure Shock Compression of Solids, (edited by J.R. Asay and M. Shahinpoor), Springer-Verlag, New York, 1993, $187 \mathrm{pp}$.

[11] E.G. Zukas, Metals Engineering Quarterly, $6(10660,16$.

[12] D.G. Doran and R.K. Linde, Solid State Physics, 19 (1966), 229.

[13] R.N. Orava and R.H. Wittman, Techniques for the Control and Application of Explosive Shock Waves, in Proceedings of the $5^{\text {th }}$ International Conference on High Energy Fabrication, University of Denver, Denver, 1975, p.1.

[14] P.S. Decarli and M.A. Meyers, Design of Uniaxial Strain Shock Recovery Experiments, in Shock Waves and High Strain Rate Phenomena in Metals, (edited by M.A. Meyers and L.E. Murr), Plenum, New York, 1981, 341 pp.

[15] A.L. Stevens and O.E. Jones, Journal of Applied Mechanics, 39 (1972), 359.

[16] O. T. Strand, et. al., Rev. Sci. Instrum. 77, 083108 (2006)

[17] D. Shechtman, Metallurgical Transactions A, 7A, pp. 151-152 (1976). 